\title{
DRIS NA AVALIAÇÃO DO ESTADO NUTRICIONAL DO ARROZ IRRIGADO POR INUNDAÇÃO(1)
}

\author{
Raquel Hermann Pötter Guindani ${ }^{(2)}$, Ibanor Anghinoni ${ }^{(3)}$ \& \\ Gilmar Ribeiro Nachtigall ${ }^{(4)}$
}

\begin{abstract}
RESUMO
O alto custo das adubações, a importância da nutrição mineral na produtividade e o nível de tecnologia adotado nas lavouras de arroz irrigado no sul do Brasil tornam relevante a utilização de técnicas de avaliação do estado nutricional, como a diagnose foliar. Entre os métodos para diagnóstico nutricional das plantas, destaca-se o Sistema Integrado de Diagnose e Recomendação (DRIS), que utiliza o conceito do balanço de nutrientes. $O$ objetivo deste trabalho foi estabelecer normas de referência DRIS e padrões nutricionais para a cultura do arroz irrigado por inundação. Foram utilizados resultados de análise foliar e de produtividade de 356 lavouras das diferentes regiões arrozeiras do Rio Grande do Sul na safra 2005/2006. As amostragens foram feitas no estádio de florescimento da cultura. A população de alta produtividade foi determinada pela melhor correlação entre o Índice de Balanço Nutricional (IBN) e a produtividade, sendo ela a que atingiu produtividade $\geq 9.000 \mathrm{~kg} \mathrm{ha}^{-1}$. O cálculo das funções e índices DRIS baseou-se no método original de Beaufils. Os resultados indicaram que: a concentração dos nutrientes mostrou relação positiva com os respectivos índices DRIS; o IBN apresentou correlação negativa com a produtividade; a determinação do ponto de equilíbrio DRIS permitiu o estabelecimento de um padrão nutricional para o arroz irrigado por inundação; $e$ o método DRIS, utilizando normas específicas para o Rio Grande do Sul, foi eficiente no diagnóstico nutricional do arroz irrigado por inundação.
\end{abstract}

Termos de indexação: diagnose foliar, índice de balanço nutricional, padrão nutricional.

\footnotetext{
(1) Parte da Dissertação de Mestrado do primeiro autor apresentada ao Programa de Pós-Graduação em Ciência do Solo, Universidade Federal do Rio Grande do Sul - UFGRS. Recebido para publicação em abril de 2008 e aprovado em dezembro de 2008.

(2) Engenheira-Agrônoma, Mestre em Ciência do Solo da Universidade Federal do Rio Grande do Sul. Rua José Bonifácio 829/ 102, CEP 96450-000 Dom Pedrito (RS). E-mail: rhpguindani@yahoo.com.br

${ }^{(3)}$ Professor Adjunto do Departamento de Solos, Universidade Federal do Rio Grande do Sul - UFRGS. Av. Bento Gonçalves 7712, Caixa Postal 776, CEP 91540-000 Porto Alegre (RS). E-mail: ibanghi@ufrgs.br

(4) Pesquisador da Embrapa Uva e Vinho. BR 285, Km 4, Caixa Postal 1513, CEP 95200-000 Vacaria (RS). E-mail: gilmar.nachtigall@terra.com.br
} 


\title{
SUMMARY: DRIS TO EVALUATE NUTRITIONAL STATUS OF FLOODED RICE
}

\begin{abstract}
In view of the high cost of fertilizers and the importance of mineral nutrition on the technology level used by rice producers for yields in southern Brazil, the use of evaluation techniques of the nutritional status, such as foliar diagnosis has become essential. Among the methods for nutritional plant diagnosis, the Diagnosis and Recommendation Integrated System (DRIS) stands out, which is based on the concept of nutrient balance. The objective of this study was to establish a DRIS reference norm and nutritional standards for flooded rice. Results of foliar analysis and grain yield of 356 farms in different rice-producing regions in the 2005/ 2006 growing season were used. The leaf samples were collected at the flowering stage. Highyielding populations were selected based on the best correlation between the Index of Nutritional Balance (IBN) and the maximum yield, which reached a more than $9.000 \mathrm{~kg} \mathrm{ha}^{-1}$. The calculation of the DRIS functions and indexes was based on the original method of Beaufils. Results indicated that: the nutrient concentration was positively related with the respective DRIS indexes; the IBN correlated negatively with yield; the determination of the DRIS equilibrium point allowed the establishment of nutritional standards for flooded rice; and the DRIS method, using specific norms for the Rio Grande do Sul State, was efficient for a nutritional diagnosis of flooded rice.
\end{abstract}

Index terms: foliar diagnosis, nutritional balance index, nutritional standards.

\section{INTRODUÇÃO}

O Brasil produz anualmente em torno de $12 \mathrm{Mt}$ de arroz (CONAB, 2008), o que lhe garante autossustentação desse alimento. Desse total, em torno de $70 \%$ é cultivado em solo alagado, especialmente nos dois Estados do extremo sul (Azambuja et al., 2004). A irrigação por inundação caracteriza-se pela manutenção de uma lâmina d‘água de aproximadamente $15 \mathrm{~cm}$ de altura sobre o solo, durante o ciclo de desenvolvimento da cultura (100-120 dias). Nesses dois Estados da região Sul, a produtividade das lavouras vem aumentando substancialmente nos últimos anos, atingindo na safra 2007/08 valores médios de $6.860 \mathrm{~kg} \mathrm{ha}^{-1}$ no Rio Grande do Sul e $7.500 \mathrm{~kg} \mathrm{ha}^{-1}$ em Santa Catarina (CONAB, 2008), fruto, essencialmente, da alteração do manejo do solo e da cultura, em que a adubação passou a exercer papel importante (Genro Jr. et al., 2007; Schoenfeld et al., 2007).

Com a elevação da produtividade de arroz e a necessidade de sua manutenção, resultado do nível de tecnologia adotado pelos produtores, e o elevado custo dos fertilizantes, demandas sobre o conhecimento da nutrição mineral dessa cultura passaram a ser evidenciadas. Assim, técnicas de avaliação do estado nutricional dela passaram a constituir ferramentas potenciais para o monitoramento da oferta e do equilíbrio entre nutrientes.

O uso da análise foliar como critério de diagnóstico baseia-se na premissa de existir relação entre o suprimento de nutrientes pelo solo e os seus teores na planta e que aumentos ou decréscimos nas concentrações dos nutrientes se relacionam com produções mais altas ou mais baixas, respectivamente (Evenhuis \& Waard, 1980). Como o teor crítico e as faixas de suficiência - critérios mais utilizados para avaliação e interpretação do estado nutricional das plantas - estão sujeitos a algumas limitações, por considerarem apenas a concentração isolada do nutriente em um determinado estádio fenológico (Hanson, 1981), o foco passou para o Sistema Integrado de Diagnose e Recomendação (DRIS). Esse método utiliza o conceito do balanço de nutrientes e parece estar menos sujeito às interferências de particularidades locais do ambiente e das variações de amostragem com respeito à idade e origem do tecido da planta. Esse sistema, proposto por Beaufils (1973), considera as relações entre os nutrientes e as compara com um padrão de alta produtividade, calculando um índice para cada nutriente e permitindo identificar quais são os elementos mais limitantes e, ou, em excesso. O DRIS utiliza as relações binárias entre os nutrientes e transforma os valores das concentrações em índices, que variam de negativo a positivo. Quanto menor for o índice, se negativo, mais limitante por deficiência estará o nutriente, e quanto maior for o índice, se positivo, mais excessivo estará o nutriente. $\mathrm{O}$ índice de valor zero indica que o nutriente está nas melhores condições de balanço nutricional (Walworth \& Sumner, 1987).

Após a definição das normas e das relações para cada par de nutrientes, é possível calcular os índices DRIS para as concentrações de nutrientes de uma amostra. Assim, o cálculo do índice DRIS depende, inicialmente, do estabelecimento dos valores-padrão ou normas, obtidos para todos os nutrientes dois a dois, em que se utiliza uma população de alta 
produtividade como população de referência (Bataglia \& Santos, 1990). Adicionalmente aos índices DRIS, é possível calcular o Índice de Balanço Nutricional (IBN), que resulta do somatório, em módulo, dos valores dos índices DRIS de cada nutriente, indicando o estado nutricional da planta. Quanto menor for o valor da soma, menor será o desequilíbrio entre nutrientes e, portanto, maior será a produtividade da cultura (Walworth \& Sumner, 1987). Vários trabalhos mostram que o IBN apresenta alta correlação negativa com a produtividade (Creste \& Nakagawa, 1997; Veloso et al., 2000; Mourão Filho et al., 2002; Silva et al., 2003; Hoogerheide, 2005).

Entre as culturas de grãos no Brasil, o método DRIS tem sido utilizado essencialmente para avaliar o estado nutricional da cultura da soja (Oliveira, 1993; Maeda, 2002; Lantmann et al., 2002; Kurihara, 2004; Hoogerheide, 2005). Para as demais culturas de grãos, poucos são os trabalhos que utilizam os princípios e o método preconizado por Beaufils (1973). Para o arroz irrigado, nem mesmo trabalhos utilizando outros métodos de diagnose foliar foram desenvolvidos para as condições de clima, solo e cultivares do País. O objetivo deste trabalho foi estabelecer normas de referência DRIS e padrões nutricionais para a cultura do arroz irrigado por inundação no Rio Grande do Sul.

\section{MATERIAL E MÉTODOS}

O trabalho foi realizado utilizando-se resultados de análise foliar e de produtividade de arroz irrigado provenientes das seis regiões arrozeiras do RS: 84 amostras da Campanha (30 58'58" S/ $54^{\circ} 40$ ' 23 ” W), 57 amostras da Depressão Central $\left(29^{\circ} 38^{\prime} 43^{\prime \prime} \mathrm{S} / 53^{\circ} 14\right.$ ' 24 "W), 52 amostras da Fronteira Oeste (29 ${ }^{\circ} 45^{\prime} 17$ " S/57 ${ }^{\circ} 05$ ' 18 ” W), 54 amostras da Planície Costeira Externa $\left(30^{\circ} 15 ' 28\right.$ ' $\mathrm{S} / 5030$ ' 35 "W), 44 amostras da Planície Costeira Interna (29 54 ' $18^{\prime \prime} \mathrm{S} /$ $51^{\circ} 45$ ' 37 "W) e 65 amostras da Região Sul $\left(32^{\circ} 02\right.$ ' 06 " $\left.\mathrm{S} / 52^{\circ} 05^{\prime} 55^{\prime} \mathrm{W}\right)$. Nessas áreas, os solos de várzea dominantes são Planossolos, Chernossolos, Gleissolos e Neossolos; os Planossolos são os que ocorrem em maior extensão. As cultivares de arroz utilizadas nas lavouras amostradas foram: BR IRGA 409, BR IRGA 410, IRGA 414, IRGA 416, IRGA 417, IRGA 418, IRGA 420, IRGA 421, IRGA 422 CL, Taim, Chuí, Qualimax 1, Qualimax 13, Arrank, Sabore, Avaxi, Tuno CL, El Passo 144, EPAGRI 108, EPAGRI 109, EPAGRI 112, EPAGRI 113 e LB 48. Dessas, a variedade de maior ocorrência nas áreas amostradas foi a IRGA 422 CL, que foi cultivada em $42,4 \%$ das lavouras, e a segunda variedade mais cultivada foi a IRGA 417, em 11,6\% das lavouras. O sistema de preparo do solo predominante foi o cultivo mínimo, e o tamanho médio das lavouras amostradas foi de 100 ha.
As amostras foliares foram coletadas na safra 2005/ 2006 em 389 lavouras comerciais do RS, com potencial de baixa, média e alta produtividade; destas, algumas tiveram de ser eliminadas por falta de dados de produtividade, restando 356 amostras. A amostragem consistiu na coleta de 50 folhas-bandeira (última folha expandida antes da emissão da panícula) de cada talhão de lavoura no início do estádio reprodutivo da cultura, isto é, quando esta se encontrava com menos de $50 \%$ de floração. Essas folhas foram coletadas de plantas que ainda não apresentavam panícula visível, embora parte das plantas da lavoura já tivesse emitido a panícula.

A colheita de grãos de arroz foi efetuada na mesma área correspondente à amostragem do tecido foliar, por máquina colhedora automotriz, após a maturação fisiológica, com a umidade entre 18 e $23 \%$, dependendo da cultivar e do sistema de colheita e de secagem (SOSBAI, 2005); foi efetuado o registro desses resultados de produtividade, com a correção da umidade para $13 \%$.

Após secagem e moagem, as folhas foram analisadas pelo Laboratório de Análises de Solo, Planta e Outros Materiais do Departamento de Solos da Faculdade de Agronomia da UFRGS, para determinação dos teores de macro e de micronutrientes, segundo método descrito em Tedesco et al. (1995).

Os resultados das análises químicas do tecido vegetal para $\mathrm{N}, \mathrm{P}, \mathrm{K}, \mathrm{Ca}, \mathrm{Mg}, \mathrm{S}, \mathrm{Cu}, \mathrm{Zn}, \mathrm{Fe}, \mathrm{Mn}, \mathrm{B}$ e Mo, juntamente com os respectivos resultados de produtividade, foram colocados em planilha eletrônica Microsoft Excel ${ }^{\mathrm{TM}}$ e organizados por região de origem. O banco de dados foi separado em uma população de alta e outra de baixa produtividade, para estabelecimento das normas DRIS. O banco de dados foi dividido em 19 classes de produtividade, com intervalo de classe de $450 \mathrm{~kg} \mathrm{ha}^{-1}$, a fim de correlacionar os índices DRIS com a produtividade.

Os cálculos das normas, dos índices DRIS, do IBN e do Balanço Nutricional médio (IBNm) foram feitos utilizando-se a planilha DRIS do software Microsoft Excel $^{\mathrm{TM}}$ desenvolvida pelo pesquisador Gilmar Ribeiro Nachtigall, da Embrapa Uva e Vinho, empregandose para os cálculos o método original proposto por Beaufils (1973). O IBN foi calculado pelo somatório dos valores absolutos dos índices gerados para a amostra, obtidos para cada nutriente, e o IBNm foi obtido pelo IBN de cada amostra dividido pelo número de nutrientes que participam do cálculo (Wadt, 1996). A norma foi estabelecida utilizando-se as concentrações dos nutrientes da população de alta produtividade, adotando-se para divisão das duas subpopulações (alta e baixa produtividade) o critério utilizado por Creste (1996) e Hoogerheide (2005), sendo escolhida a população na qual a norma oriunda de determinado nível de produtividade apresentasse a melhor relação entre o IBN e a produtividade. O critério usado para determinar a produtividade de separação entre as populações de alta e de baixa produtividade foi baseado 
no nível de produtividade considerado alto de acordo com a média alcançada pelos produtores de arroz irrigado no RS. Assim, adotou-se a produtividade de $7.650 \mathrm{~kg} \mathrm{ha}^{-1}$ como limite entre as duas subpopulações. A partir dessa produtividade, foram estabelecidas as classes de população de alta produtividade: $\geq 7.650 \mathrm{~kg} \mathrm{ha}^{-1} ; \geq 8.100 \mathrm{~kg} \mathrm{ha}^{-1} ; \geq 8.550 \mathrm{~kg} \mathrm{ha}^{-1} ; \mathrm{e}$ $\geq 9.000 \mathrm{~kg} \mathrm{ha}^{-1}$.

Dentro da população de cada classe de produtividade estabeleceu-se a população nutricionalmente equilibrada (Beaufils, 1956, citado por Beaufils, 1971), que é considerada aquela cujas concentrações de nutrientes estejam dentro do limite da média mais ou menos dois terços do desvio-padrão. Para as quatro populações consideradas de alta produtividade foram calculadas as normas, os índices DRIS e o IBN para estudo da relação com a produtividade. Foi selecionada, então, a norma que apresentou a melhor relação entre IBN e produtividade entre as populações de referência testadas.

A decisão de usar no cálculo das normas a relação direta ou inversa da razão de nutrientes foi feita pelo método da razão das variâncias valor $\mathrm{F}$, descrito por Letzsch (1985) e Walworth et al. (1986). Esse critério consiste no cálculo da razão de variância das relações entre nutrientes da subpopulação de referência (r) e de baixa produtividade (b), tanto na relação direta quanto inversa. Foi escolhida a ordem da relação que apresentou a maior razão de variância entre a população de alta e a de baixa produtividade.

As funções das relações dos nutrientes e os índices DRIS foram calculadas utilizando-se o método original proposto por Beaufils (1973). O IBN foi calculado pelo somatório dos valores absolutos dos índices gerados para a amostra, obtidos para cada nutriente, e o Índice de Balanço Nutricional médio foi obtido pelo IBN de cada amostra dividido pelo número de nutrientes que participam do cálculo (Wadt, 1996).
Para cada um dos nutrientes analisados, foram estabelecidas três classes nutricionais, considerando o índice DRIS do nutriente e o IBNm: deficiente, adequado e excessivo, seguindo o método proposto por Wadt (1996). O nutriente foi considerado deficiente quando apresentou índice DRIS negativo e, em módulo, mostrou valor maior que o IBNm; foi considerado adequado quando apresentou índice DRIS negativo ou positivo, porém em módulo menor que o IBNm; e excessivo quando o índice DRIS foi positivo, e, em módulo, o valor foi maior que o IBNm.

\section{RESULTADOS E DISCUSSÃO}

As concentrações de macro e de micronutrientes nas folhas analisadas apresentaram distribuição próxima à normalidade (Quadro 1), a exemplo do observado por Hoogerheide (2005) na cultura da soja. As relações entre a concentração de nutrientes e a produtividade apresentaram baixa correlação, evidenciando a maior importância do balanço de nutrientes na determinação da produtividade do arroz irrigado do que a própria concentração de cada nutriente. Mesmo com o agrupamento dos dados por classe de produtividade, não foi possível verificar relação clara e direta entre produtividade de grãos e concentração de nutrientes (Guindani, 2007).

Observa-se (Figura 1) que houve relação significativa $(p<0,01)$ entre a produtividade e o IBN para todas as faixas de produtividade e que a norma obtida com a população de referência de produtividade $\geq 9.000 \mathrm{~kg} \mathrm{ha}^{-1}$ foi a que apresentou maior relação entre essas variáveis $\left(\mathrm{R}^{2}=0,73\right)$, sendo, então, adotada para os cálculos da norma DRIS para o arroz irrigado (Quadro 2).

O coeficiente de determinação obtido para a relação entre o IBN e a produtividade na população de

Quadro 1. Estatísticas descritivas das concentrações de macro e de micronutrientes no tecido foliar de arroz irrigado em lavouras do Rio Grande do Sul, safra 2005/2006, estádio R2-R3

\begin{tabular}{|c|c|c|c|c|c|c|c|c|c|c|}
\hline Nutriente & Média & Mediana & Moda & Mín. & Máx. & Desvio-padrão & Variância & CV (\%) & Curtose & Assimetria \\
\hline $\mathrm{N}\left(\mathrm{g} \mathrm{kg}^{-1}\right)$ & 25,1 & 25 & 24 & 17 & 40 & 3,3 & 10,9 & 13,1 & 1,7 & 0,8 \\
\hline $\mathrm{P}\left(\mathrm{g} \mathrm{kg}^{-1}\right)$ & 2,1 & 2,1 & 2,2 & 1 & 3,2 & 0,3 & 0,1 & 16,1 & 0,6 & $-0,1$ \\
\hline $\mathrm{K}\left(\mathrm{g} \mathrm{kg}^{-1}\right)$ & 12,6 & 13 & 13 & 5,4 & 21 & 2,4 & 5,9 & 19,3 & 0,5 & 0,1 \\
\hline $\mathrm{Ca}\left(\mathrm{g} \mathrm{kg}^{-1}\right)$ & 3,0 & 2,9 & 2,7 & 1,3 & 8,3 & 0,9 & 0,9 & 30,8 & 3,1 & 1,2 \\
\hline $\mathrm{Mg}\left(\mathrm{g} \mathrm{kg}^{-1}\right)$ & 1,5 & 1,5 & 1,7 & 0,7 & 3,2 & 0,4 & 0,1 & 24,7 & 1,4 & 0,7 \\
\hline $\mathrm{S}\left(\mathrm{g} \mathrm{kg}^{-1}\right)$ & 1,9 & 1,8 & 1,8 & 1,0 & 3,6 & 0,30 & 0,1 & 17,5 & 4,4 & 1,3 \\
\hline $\mathrm{Cu}\left(\mathrm{mg} \mathrm{kg}^{-1}\right)$ & 4,5 & 4 & 4 & 2 & 15 & 1,5 & 2,2 & 32,8 & 7,7 & 1,7 \\
\hline $\mathrm{Zn}\left(\mathrm{mg} \mathrm{kg}^{-1}\right)$ & 19 & 19 & 19 & 9 & 102 & 6,6 & 43,8 & 34,8 & 77,4 & 6,9 \\
\hline $\mathrm{Fe}\left(\mathrm{mg} \mathrm{kg}{ }^{-1}\right)$ & 116 & 100 & 83 & 44 & 691 & 68,2 & 4649,1 & 58,8 & 29,6 & 4,5 \\
\hline $\mathrm{Mn}\left(\mathrm{mg} \mathrm{kg}^{-1}\right)$ & 558 & 429 & 1.100 & 55 & 3.000 & 460,3 & $211.832,3$ & 82,4 & 5,3 & 2 \\
\hline $\mathrm{B}\left(\mathrm{mg} \mathrm{kg}{ }^{-1}\right)$ & 6,9 & 6 & 5 & 1 & 27 & 4 & 15,7 & 57,6 & 3,2 & 1,4 \\
\hline Mo (mg kg-1) & 1,2 & 1,1 & 1 & 0,2 & 4,5 & 0,6 & 0,4 & 50,5 & 4,4 & 1,5 \\
\hline
\end{tabular}



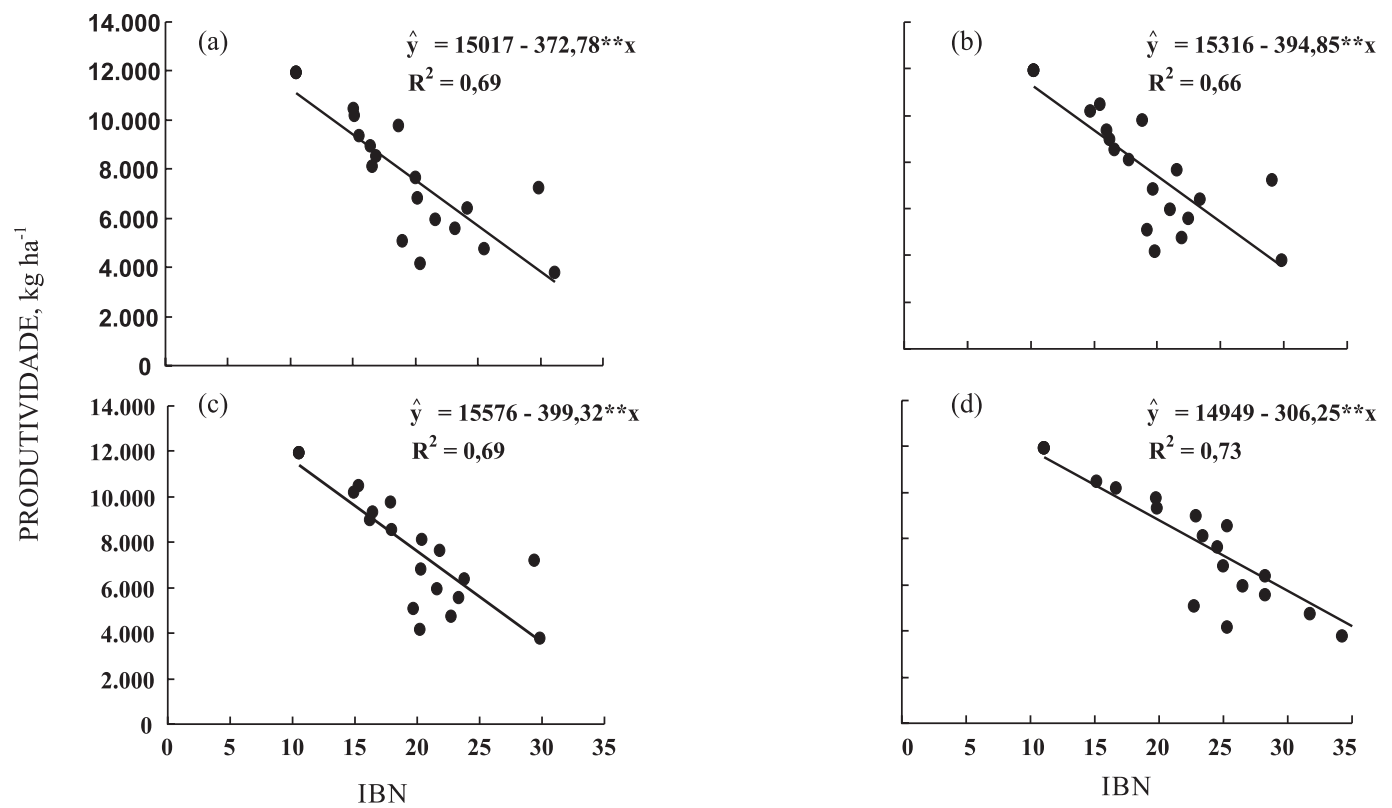

Figura 1. Relação entre o Índice de Balanço Nutricional (IBN) e a produtividade em diferentes faixas de produtividade da subpopulação de referência: $\geq 7.650 \mathrm{~kg} \mathrm{ha}^{-1}$ (a); $8.100 \mathrm{~kg} \mathrm{ha}^{-1}$ (b); $\geq 8.550 \mathrm{~kg} \mathrm{ha}^{-1}$ (c); e $\geq 9.000 \mathrm{~kg} \mathrm{ha}^{-1}$ (d) para o arroz irrigado por inundação do Rio Grande do Sul, safra 2005/2006.

Quadro 2. Norma DRIS para a cultura do arroz irrigado por inundação do Rio Grande do Sul, safra 2005/2006

\begin{tabular}{|c|c|c|c|c|c|c|c|c|c|}
\hline$N^{\circ}$ de ordem & Relação & Média & $\mathrm{CV}^{(1)}$ & $\mathbf{S}^{(2)}$ & $N^{\circ}$ de ordem & Relação & Média & $\mathrm{CV}^{(1)}$ & $\mathrm{S}^{(2)}$ \\
\hline 1 & $\mathrm{~N} / \mathrm{P}$ & 12,52 & 9,73 & 1,22 & 34 & $\mathrm{Ca} / \mathrm{Cu}$ & 0,66 & 23,90 & 0,16 \\
\hline 2 & $\mathrm{~N} / \mathrm{K}$ & 2,13 & 16,22 & 0,35 & 35 & $\mathrm{Fe} / \mathrm{Ca}$ & 40,17 & 43,17 & 17,34 \\
\hline 3 & $\mathrm{Ca} / \mathrm{N}$ & 0,10 & 21,97 & 0,02 & 36 & $\mathrm{Mn} / \mathrm{Ca}$ & 191,19 & 43,13 & 82,47 \\
\hline 4 & $\mathrm{Mg} / \mathrm{N}$ & 0,05 & 21,07 & 0,01 & 37 & $\mathrm{Ca} / \mathrm{Mo}$ & 2,32 & 53,49 & 1,24 \\
\hline 5 & $\mathrm{~S} / \mathrm{N}$ & 0,07 & 12,65 & 0,01 & 38 & $\mathrm{Ca} / \mathrm{Zn}$ & 0,15 & 30,71 & 0,05 \\
\hline 6 & $\mathrm{~B} / \mathrm{N}$ & 0,28 & 53,09 & 0,15 & 39 & $\mathrm{Mg} / \mathrm{S}$ & 0,79 & 21,01 & 0,17 \\
\hline 7 & $\mathrm{~N} / \mathrm{Cu}$ & 6,41 & 25,14 & 1,61 & 40 & $\mathrm{Mg} / \mathrm{B}$ & 0,28 & 78,09 & 0,22 \\
\hline 8 & $\mathrm{Fe} / \mathrm{N}$ & 3,88 & 29,21 & 1,13 & 41 & $\mathrm{Mg} / \mathrm{Cu}$ & 0,34 & 26,19 & 0,09 \\
\hline 9 & $\mathrm{Mn} / \mathrm{N}$ & 19,76 & 42,74 & 8,45 & 42 & $\mathrm{Fe} / \mathrm{Mg}$ & 72,91 & 34,89 & 25,43 \\
\hline 10 & N/Mo & 22,53 & 46,01 & 10,36 & 43 & $\mathrm{Mn} / \mathrm{Mg}$ & 359,11 & 41,02 & 147,32 \\
\hline 11 & $\mathrm{Zn} / \mathrm{N}$ & 0,72 & 20,85 & 0,15 & 44 & $\mathrm{Mg} / \mathrm{Mo}$ & 1,21 & 51,72 & 0,63 \\
\hline 12 & $\mathrm{~K} / \mathrm{P}$ & 6,02 & 14,45 & 0,87 & 45 & $\mathrm{Zn} / \mathrm{Mg}$ & 13,79 & 21,77 & 3,00 \\
\hline 13 & $\mathrm{Ca} / \mathrm{P}$ & 1,29 & 21,04 & 0,27 & 46 & $\mathrm{~B} / \mathrm{S}$ & 4,15 & 54,86 & 2,28 \\
\hline 14 & $\mathrm{Mg} / \mathrm{P}$ & 0,68 & 19,59 & 0,13 & 47 & $\mathrm{~S} / \mathrm{Cu}$ & 0,44 & 21,39 & 0,09 \\
\hline 15 & $\mathrm{~S} / \mathrm{P}$ & 0,86 & 12,35 & 0,11 & 48 & $\mathrm{Fe} / \mathrm{S}$ & 56,43 & 34,85 & 19,66 \\
\hline 16 & $\mathrm{~B} / \mathrm{P}$ & 3,46 & 54,91 & 1,90 & 49 & $\mathrm{Mn} / \mathrm{S}$ & 283,10 & 44,65 & 126,40 \\
\hline 17 & $\mathrm{P} / \mathrm{Cu}$ & 0,52 & 23,14 & 0,12 & 50 & S/Mo & 1,55 & 49,49 & 0,77 \\
\hline 18 & $\mathrm{Fe} / \mathrm{P}$ & 50,73 & 37,91 & 19,23 & 51 & $\mathrm{Zn} / \mathrm{S}$ & 10,49 & 18,42 & 1,93 \\
\hline 19 & $\mathrm{Mn} / \mathrm{P}$ & 245,88 & 43,14 & 106,08 & 52 & $\mathrm{~B} / \mathrm{Cu}$ & 1,72 & 53,04 & 0,91 \\
\hline 20 & $\mathrm{P} / \mathrm{Mo}$ & 1,77 & 41,04 & 0,73 & 53 & $\mathrm{Fe} / \mathrm{B}$ & 20,56 & 78,05 & 16,05 \\
\hline 21 & $\mathrm{Zn} / \mathrm{P}$ & 9,03 & 19,12 & 1,73 & 54 & $\mathrm{~B} / \mathrm{Mn}$ & 0,02 & 109,86 & 0,02 \\
\hline 22 & $\mathrm{Ca} / \mathrm{K}$ & 0,22 & 29,23 & 0,06 & 55 & $\mathrm{~B} / \mathrm{Mo}$ & 6,47 & 71,22 & 4,61 \\
\hline 23 & $\mathrm{Mg} / \mathrm{K}$ & 0,11 & 19,89 & 0,02 & 56 & $\mathrm{~B} / \mathrm{Zn}$ & 0,40 & 57,96 & 0,23 \\
\hline 24 & $\mathrm{~S} / \mathrm{K}$ & 0,14 & 16,54 & 0,02 & 57 & $\mathrm{Fe} / \mathrm{Cu}$ & 24,34 & 37,21 & 9,06 \\
\hline 25 & $\mathrm{~B} / \mathrm{K}$ & 0,57 & 52,76 & 0,30 & 58 & $\mathrm{Mn} / \mathrm{Cu}$ & 118,63 & 39,13 & 46,42 \\
\hline 26 & $\mathrm{Cu} / \mathrm{K}$ & 0,35 & 27,94 & 0,10 & 59 & $\mathrm{Cu} / \mathrm{Mo}$ & 3,93 & 76,02 & 2,99 \\
\hline 27 & $\mathrm{Fe} / \mathrm{K}$ & 8,60 & 40,17 & 3,45 & 60 & $\mathrm{Zn} / \mathrm{Cu}$ & 4,57 & 28,01 & 1,28 \\
\hline 28 & $\mathrm{Mn} / \mathrm{K}$ & 42,72 & 50,35 & 21,51 & 61 & $\mathrm{Fe} / \mathrm{Mn}$ & 0,26 & 90,54 & 0,23 \\
\hline 29 & $\mathrm{~K} / \mathrm{Mo}$ & 10,77 & 45,49 & 4,90 & 62 & $\mathrm{Fe} / \mathrm{Mo}$ & 85,12 & 46,61 & 39,68 \\
\hline 30 & $\mathrm{Zn} / \mathrm{K}$ & 1,50 & 16,16 & 0,24 & 63 & $\mathrm{Fe} / \mathrm{Zn}$ & 5,67 & 40,82 & 2,32 \\
\hline 31 & $\mathrm{Ca} / \mathrm{Mg}$ & 1,97 & 22,71 & 0,45 & 64 & $\mathrm{Mn} / \mathrm{Mo}$ & 448,75 & 71,66 & 321,56 \\
\hline 32 & $\mathrm{Ca} / \mathrm{S}$ & 1,52 & 21,91 & 0,33 & 65 & $\mathrm{Mn} / \mathrm{Zn}$ & 29,07 & 57,05 & 16,58 \\
\hline 33 & $\mathrm{~B} / \mathrm{Ca}$ & 2,69 & 51,94 & 1,40 & 66 & $\mathrm{Zn} / \mathrm{Mo}$ & 16,04 & 44,44 & 7,13 \\
\hline
\end{tabular}

(1) CV: coeficiente de variação (\%). ${ }^{(2)} \mathrm{s}$ : desvio-padrão. 
referência escolhida $\left(R^{2}=0,73\right)$ representa um valor satisfatório para o arroz irrigado, visto que essa é uma cultura cujo estado nutricional é de difícil correlação com os dados de produtividade e outros componentes da produção, devido à sua peculiar condição de irrigação por inundação, que altera substancialmente as características químicas, físicas e biológicas do solo. Para a cultura da soja, Hoogerheide (2005) obteve coeficientes de determinação de 0,91 (Maranhão e Piauí) e de 0,86 (Mato Grosso); já para limoeiro, Creste (1996) obteve $\mathrm{R}^{2}$ de somente 0,58 .

Do total das 356 amostras analisadas, 31 fizeram parte da norma DRIS, representando $9 \%$ do banco de dados total, o que é aceitável, pois Letzsch \& Sumner (1984) afirmam que a população de referência deve conter em torno de $10 \%$ das observações do banco de dados.

Definidas as normas, foram calculados os índices DRIS; verificaram-se, então, as relações positivas e significativas $(p<0,01)$ entre as concentrações de todos os nutrientes e os respectivos índices DRIS (Figuras 2 e 3). No caso de $\mathrm{Ca}, \mathrm{Mg}, \mathrm{Cu}, \mathrm{Zn}, \mathrm{Fe}, \mathrm{Mn}, \mathrm{B}$ e Mo, os coeficientes de determinação foram mais elevados $\left(\mathrm{R}^{2}>0,50\right)$, indicando que o índice DRIS foi dependente da concentração do próprio nutriente na folha. Já para N, P, K e S houve baixa relação entre essas variáveis, o que indica menor dependência dos índices
DRIS desses nutrientes em relação à sua concentração foliar, demonstrando maior dependência das concentrações dos demais nutrientes que compõem o índice DRIS, ou seja, para esses nutrientes a influência do balanço nutricional na formação do índice DRIS é maior, em relação aos demais.

É importante atentar para as curvas dos micronutrientes $\mathrm{Mn}, \mathrm{B}$ e Mo, que, diferentemente dos demais nutrientes, apresentaram elevado ajuste logarítmico da regressão (Figura 3), o que demonstra que o método DRIS está superestimando as deficiências dos nutrientes em questão. Assim, esses resultados devem ser analisados com cautela, visto que existe forte indicação de se considerar a sensibilidade do método para esses micronutrientes em termos de equilíbrio nutricional. Segundo Maia (1999), a fórmula original de Beaufils (1973), utilizada neste trabalho, superestima a deficiência nutricional quando a relação binária entre nutrientes é menor que a relação da população de referência, o que aconteceu em parte das amostras deste trabalho. Wadt et al. (2007) inferiram que os nutrientes Mn, B e Mo apresentaram pequena amplitude para valores de concentração considerados ótimos. Desse modo, se fosse aplicada, segundo esses autores, uma forma de correção ao cálculo das funções DRIS desses micronutrientes, valores mais amplos dessas funções poderiam ser definidos à medida que o valor da relação
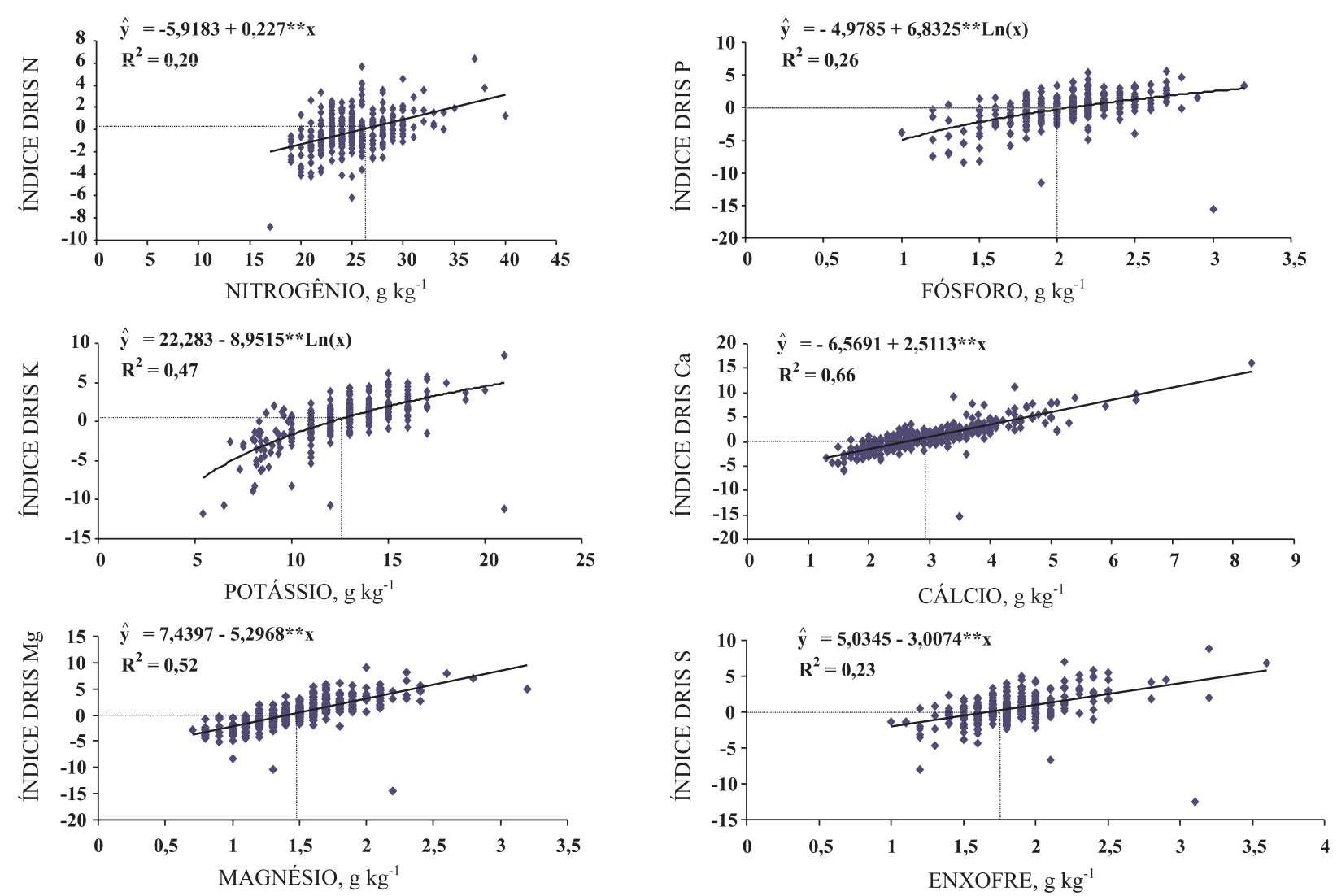

Figura 2. Relação entre os índices DRIS e a concentração de macronutrientes nas folhas de lavouras de arroz irrigado por inundação do Rio Grande do Sul, safra 2005/2006. 

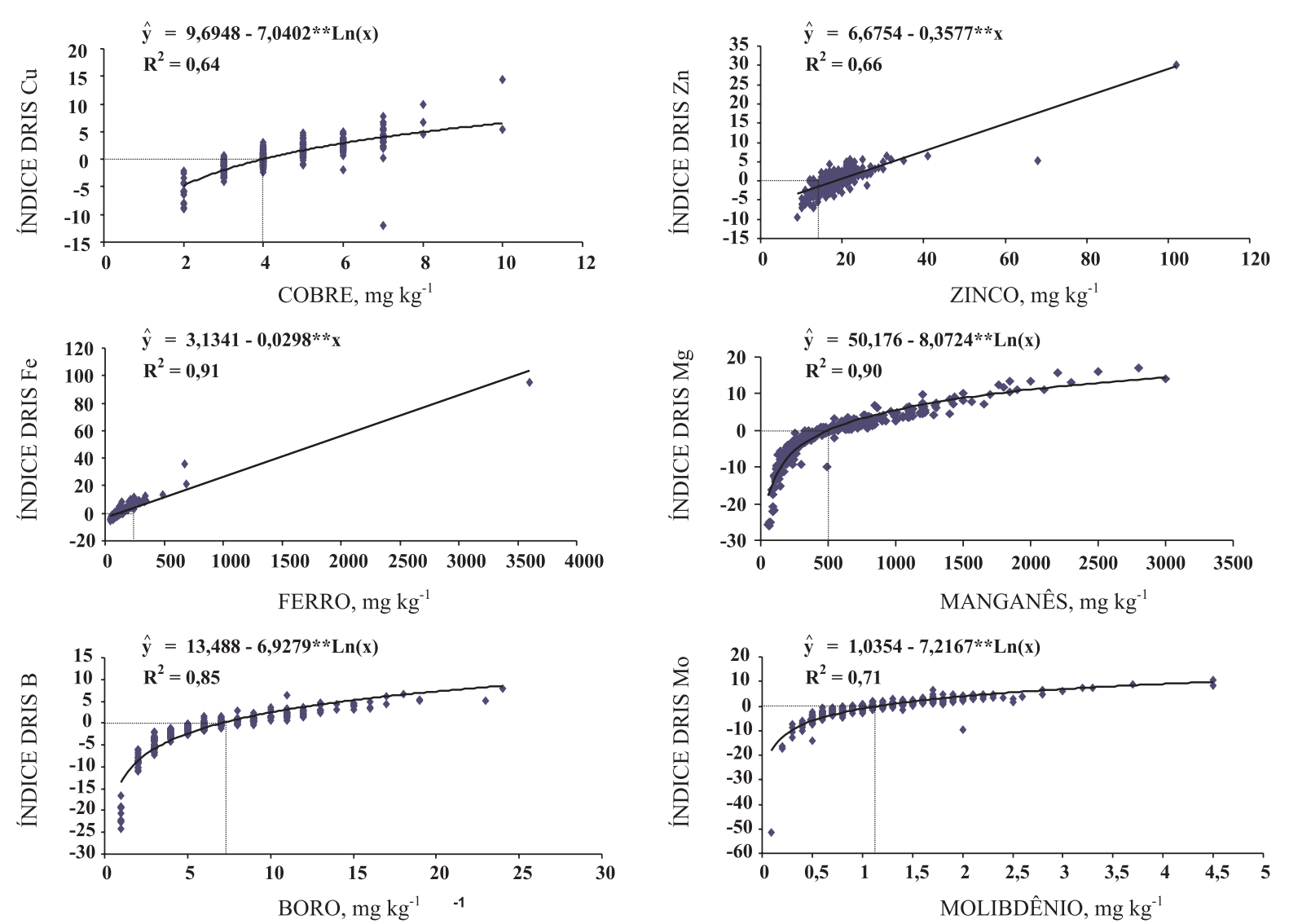

Figura 3. Relação entre os índices DRIS e a concentração de micronutrientes nas folhas de lavouras de arroz irrigado por inundação do Rio Grande do Sul, safra 2005/2006.

entre dois nutrientes se afastasse do valor ótimo para a relação.

Levando-se em conta que a planta está equilibrada nutricionalmente quando os valores dos índices DRIS estão mais próximos de zero (Walworth \& Summer, 1987), estabeleceram-se graficamente as concentrações dos nutrientes na folha equivalentes a esse ponto de equilíbrio nutricional (Figuras 2 e 3), que são apresentadas no quadro 3 .

No quadro 4 é apresentada a distribuição de frequência das amostras foliares de lavouras de arroz irrigado por inundação do Rio Grande do Sul (safra 2005/2006), em classes nutricionais definidas pelo método DRIS, conforme o critério de Wadt (1996).

Observa-se (Quadro 4) que a grande maioria dos nutrientes situou-se, em maior percentual, na classe adequada de diagnose. As exceções foram o Mn e o B. O Mn apresentou deficiência em $40 \%$ dos casos, o que evidencia um mascaramento dos índices DRIS desse nutriente em relação aos outros, em razão da alta tolerância da planta de arroz às concentrações elevadas de Mn na folha, em decorrência da redução do solo devido ao alagamento, e da superestimação da deficiência desse nutriente pelo DRIS, mostrada pela falta de linearidade do ajuste (Figura 3). Já com relação ao B, os dados mostram que em 34 \% dos casos
Quadro 3. Ponto de equilíbrio nutricional DRIS para os nutrientes na cultura do arroz irrigado por inundação do Rio Grande do Sul, safra 2005/2006

\begin{tabular}{lc}
\hline Nutriente & $\begin{array}{c}\text { Ponto de equilíbrio } \\
\text { nutricional DRIS }\end{array}$ \\
\hline $\mathrm{N}\left(\mathrm{g} \mathrm{kg}^{-1}\right)$ & 26 \\
$\mathrm{P}\left(\mathrm{g} \mathrm{kg}^{-1}\right)$ & 2,0 \\
$\mathrm{~K}\left(\mathrm{~g} \mathrm{~kg}^{-1}\right)$ & 12,5 \\
$\mathrm{Ca}\left(\mathrm{g} \mathrm{kg}^{-1}\right)$ & 2,9 \\
$\mathrm{Mg}\left(\mathrm{g} \mathrm{kg}^{-1}\right)$ & 1,5 \\
$\mathrm{~S}\left(\mathrm{~g} \mathrm{~kg}^{-1}\right)$ & 1,8 \\
$\mathrm{Cu}\left(\mathrm{mg} \mathrm{kg}^{-1}\right)$ & 4 \\
$\mathrm{Zn}\left(\mathrm{mg} \mathrm{kg}^{-1}\right)$ & 18 \\
$\mathrm{Fe}\left(\mathrm{mg} \mathrm{kg}^{-1}\right)$ & 100 \\
$\mathrm{Mn}\left(\mathrm{mg} \mathrm{kg}^{-1}\right)$ & 500 \\
$\mathrm{~B}\left(\mathrm{mg} \mathrm{kg}^{-1}\right)$ & 7 \\
$\mathrm{Mo}\left(\mathrm{mg} \mathrm{kg}^{-1}\right)$ & 1,2 \\
\hline
\end{tabular}

(1) Concentração do nutriente nas folhas de plantas nutricionalmente equilibradas.

foi diagnosticada a sua deficiência, evidenciando sua possível limitação à produtividade. No entanto, é importante ressaltar que houve uma superestimação, 
Quadro 4. Distribuição de frequência das amostras foliares de arroz irrigado por inundação do Rio Grande do Sul (safra 2005/2006) em classes nutricionais definidas pelo método DRIS

\begin{tabular}{|c|c|c|c|c|c|c|}
\hline \multirow{3}{*}{ Nutriente } & \multicolumn{6}{|c|}{ Classe nutricional } \\
\hline & \multicolumn{2}{|c|}{ Deficiente } & \multicolumn{2}{|c|}{ Adequado } & \multicolumn{2}{|c|}{ Excessivo } \\
\hline & $\mathrm{n}^{(1)}$ & $\%$ & $\mathrm{n}^{(1)}$ & $\%$ & $\mathrm{n}^{(1)}$ & $\%$ \\
\hline $\mathrm{N}$ & 52 & 15 & 274 & 79 & 20 & 6 \\
\hline $\mathrm{P}$ & 46 & 13 & 274 & 77 & 35 & 10 \\
\hline $\mathrm{K}$ & 48 & 14 & 222 & 63 & 85 & 24 \\
\hline $\mathrm{Ca}$ & 39 & 11 & 202 & 57 & 113 & 32 \\
\hline $\mathrm{Mg}$ & 54 & 15 & 199 & 56 & 100 & 28 \\
\hline $\mathrm{S}$ & 21 & 6 & 289 & 82 & 43 & 12 \\
\hline $\mathrm{Cu}$ & 39 & 11 & 227 & 64 & 87 & 25 \\
\hline $\mathrm{Zn}$ & 85 & 24 & 202 & 57 & 67 & 19 \\
\hline $\mathrm{Fe}$ & 73 & 21 & 228 & 64 & 54 & 15 \\
\hline $\mathrm{Mn}$ & 142 & 40 & 118 & 33 & 94 & 27 \\
\hline $\mathrm{B}$ & 116 & 34 & 170 & 49 & 58 & 17 \\
\hline Mo & 73 & 21 & 193 & 55 & 86 & 24 \\
\hline
\end{tabular}

(1) Número de amostras.

pelo método DRIS, das deficiências desse nutriente (Figura 3) e que a indicação dessas deficiências pode não ser real, devendo ser estudado um ajuste no fator de sensibilidade no cálculo das funções DRIS.

Com o cálculo dos índices DRIS, é possível ordenar todos os nutrientes analisados, em cada uma das amostras, do mais deficiente ao mais em excesso. Assim, no quadro 5 são sapresentados os quatro nutrientes que apareceram mais vezes como os mais deficientes nas 356 amostras analisadas, de acordo com os índices DRIS calculados para elas. O Mn aparece novamente como o nutriente mais limitante, seguido, em ordem decrescente, pelo B, Mo e Zn.

Embora os resultados deste trabalho mostrem um diagnóstico de deficiência de alguns micronutrientes, como B, Mo e Zn, em parte das amostras de lavouras de arroz irrigado por inundação do Rio Grande do Sul, esse fato não pode ser generalizado, pois não significa que esteja ocorrendo deficiência desses micronutrientes de modo geral nas lavouras de arroz do Estado, já que o método DRIS desenvolvido neste trabalho pode estar superestimando essas deficiências, como discutido anteriormente. Assim, não se pode afirmar, a partir desses resultados, que adubações com micronutrientes sejam recomendadas para a cultura do arroz, pois esse é um tema que necessita de pesquisas específicas no sentido de sua constatação ou não. Segundo Scivittaro \& Machado (2004), há evidência de que os solos da região Sul do País sejam, em geral, bem providos de micronutrientes, pois os experimentos realizados até o momento não mostraram resposta positiva à sua aplicação na cultura do arroz irrigado por inundação.

Assim, a avaliação do estado nutricional do arroz irrigado por inundação no Rio Grande do Sul pelo método DRIS, feita neste trabalho, constitui-se em
Quadro 5. Nutrientes mais limitantes por deficiência à produtividade do arroz irrigado por inundação no Rio Grande do Sul, safra 2005/2006

\begin{tabular}{lrr}
\hline Nutriente & Número de casos & $\%$ \\
\hline Mn & 103 & 29 \\
B & 67 & 19 \\
Mo & 32 & 9 \\
Zn & 28 & 8 \\
\hline
\end{tabular}

uma forma de diagnóstico pontual e não em uma recomendação. Fica registrada aqui a necessidade de realização de estudos posteriores a este trabalho, para que se possa testar e comprovar (ou não) esse diagnóstico.

Com o estabelecimento das normas DRIS a partir do banco de dados estudado, torna-se viável a utilização da diagnose foliar como método de avaliação do estado nutricional de lavouras de arroz irrigado por inundação, proporcionando que cada lavoura analisada tenha seu diagnóstico, podendo este ser de suficiência, de deficiência ou de excesso de algum(ns) nutriente(s).

\section{CONCLUSÕES}

1. O método DRIS, utilizando normas estabelecidas a partir de dados de lavouras comerciais do Rio Grande do Sul, é eficiente na avaliação do estado nutricional do arroz irrigado. 
2. A concentração dos nutrientes apresenta relação positiva com os respectivos índices DRIS, e o Índice de Balanço Nutricional (IBN), calculado a partir das normas geradas, apresenta correlação negativa com a produtividade.

3. A determinação do ponto de equilíbrio DRIS permite o estabelecimento de um padrão nutricional para o arroz irrigado por inundação, viabilizando a utilização da diagnose foliar como ferramenta de monitoramento do estado nutricional da cultura.

\section{AGRADECIMENTO}

Ao Instituto Rio-Grandense do Arroz - IRGA, pelo apoio financeiro, técnico e operacional a este trabalho.

\section{LITERATURA CITADA}

AZAMBUJA, I.H.V.; VERNETTI Jr., F.J. \& MAGALHÃES Jr., A.M. Aspectos socioeconômicos da produção de arroz. In: GOMES, A.S. \& MAGALHÃES Jr., A.M. Arroz irrigado no Sul do Brasil. Brasília, Embrapa Informação Tecnológica, 2004. p.23-44

BATAGLIA, O.C. \& SANTOS, W.R. Efeito do procedimento de cálculo e da população de referência nos índices do sistema integrado de diagnose e recomendação (DRIS). R. Bras. Ci. Solo, 14:339-344, 1990.

BEAUFILS, E.R. Phisiological diagnosis - A guide for improving maize production based on principles developed for rubber trees. Fert. Soc. South African J., 1:1-30, 1971.

BEAUFILS, E.R. Diagnosis and Recomendation Integrated System (DRIS). A general scheme for experimentation and calibration based on principles developed from research in plant nutrition. Pietermeritzburg, University of Natal, 1973. 132p. (Soil Science Bulletin, 1)

CRESTE, J.E. Uso do DRIS na avaliação do estado nutricional do Limoeiro Siciliano. Botucatu, Universidade Estadual de São Paulo, 1996. 120p. (Tese de Doutorado)

CRESTE, J.E. \& NAKAGAWA, J. Estabelecimento do método DRIS para a cultura do limoeiro em função da análise foliar; 1: Cálculo das normas. R. Bras. Frutic., 19:297-305, 1997.

COMPANHIA BRASILEIRA DE ABASTECIMENTO CONAB. $6^{\circ}$ Levantamento de grãos 2007/2008. Disponível em: <www.conab.gov.br/conabweb/download/safra/ estudo_safra.pdf.> Acesso em 31 mar. 2008.

EVENHUIS, B. \& WAARD, P.W.F. Principles and practices in plant analysis. In: FAO. Soils. Rome, 1980. p.152-163. (FAO Bulletin, 38/1)

GENRO JR, S.A.; SCHOENFELD, R.; MACEDO, V.R.M.; MARCOLIN, E.; MENEZES, V.G. \& ANGHINONI, I. Eficácia das atuais recomendações de adubação para o arroz irrigado no Rio Grande do Sul.. In: CONGRESSO BRASILEIRO DO ARROZ IRRIGADO, 5., Pelotas, 2007. Anais. Pelotas, SOSBAI, 2007. v.1. p.624-626.
GUINDANI, R.H.P. DRIS para avaliação do estado nutricional do arroz irrigado no Rio Grande do Sul. Porto Alegre, Universidade Federal do Rio Grande do Sul, 2007. 94p. (Tese de Mestrado)

HANSON, R.G. DRIS evaluation on N, P, K status of determinants soybeans in Brazil. Comm. Soil Sci. Plant Anal., 12, 933-948, 1981.

HOOGERHEIDE, H.C. DRIS para avaliação do estado nutricional da soja em duas regiões do cerrado brasileiro. Piracicaba, Universidade de São Paulo, 2005. 94p. (Tese de Mestrado)

KURIHARA, C.H. Demanda de nutrientes pela soja e diagnose de seu estado nutricional. Viçosa, MG, Universidade Federal de Viçosa, 2004. 101p. (Tese de Doutorado).

LANTMANN, A.F.; PEREIRA, L.R.; ZOBIOLE, L.H.S.; CASTRO, C. \& ORTIZ, F.R. Normas DRIS regionalizadas para avaliação do balanço nutricional da soja no Estado do Paraná. In: REUNIÃO DE PESQUISA DE SOJA DA REGIÃO CENTRAL DO BRASIL, 24., São Pedro, 2002. Resumos. Londrina, Embrapa Soja, 2002. 128p.

LETZSCH, W.S. Computer program for selection of norms for use in the diagnosis and recommendation integrated system (DRIS). Comm. Soil Sci. Plant Anal., 16:339-347, 1985.

LETZSCH, W.S. \& SUMNER, M.E. Effect of population size and yield level in selection of diagnosis and recommendation integrated system (DRIS) norms. Comm. Soil Sci. Plant Anal., 15:997-1006, 1984.

MAEDA, S. Interpretação do estado nutricional de soja pelo DRIS no Mato Grosso do Sul. Curitiba, Universidade Federal do Paraná, 2002. 107p. (Tese de Doutorado)

MAIA, C. Análise crítica da fórmula original de Beaufils no cálculo dos índices DRIS: A constante de sensibilidade. In: WADT, P.G.S. \& MALAVOLTA, E., orgs. Monitoramento nutricional para a recomendação de adubação de culturas. Piracicaba, Potafós, 1999. CD-ROM.

MOURÃO FILHO, F.A.A. \& AZEVEDO, J.C. Funções e ordem da razão dos nutrientes no estabelecimento de normas DRIS em laranjeiras "Valência". Pesq. Agropec. Bras., 37:185-192, 2002.

OLIVEIRA, S.A. Avaliação do balanço nutricional no sistema solo-planta. In: CONGRESSO BRASILEIRO DE CIÊNCIA DO SOLO, 24., Goiânia, 1993. Resumos. Goiânia, Sociedade Brasileira de Ciência do Solo, 1993. p.43-33.

SCIVITTARO, W.B. \& MACHADO, M.O. Adubação e calagem para a cultura do arroz irrigado. In: GOMES, A.S. \& MAGALHÃES Jr., A.M. Arroz irrigado no sul do Brasil. Brasília, Embrapa Informação Tecnológica, 2004. p.259303.

SCHOENFELD, R.; GENRO JR, S.A.; MARCOLIN, E.; MACEDO, V.M. \& ANGHINONI, I. Estratégia de adubação para incremento de produtividade do arroz irrigado no Rio Grande do Sul. In: CONGRESSO BRASILEIRO DO ARROZ IRRIGADO, 5., Pelotas, 2007. Anais. Pelotas, SOSBAI, 2007. v.1. p.555-557. 
SILVA, E.B.; NOGEURA, F.D. \& GUIMARÃES, P.T.G. Uso do DRIS na avaliação do estado nutricional do cafeeiro em resposta à adubação potássica. R. Bras. Ci. Solo. 27:247$255,2003$.

SOCIEDADE BRASILEIRA DO ARROZ IRRIGADO - SOSBAI. Arroz irrigado - Recomendações técnicas da pesquisa para o Sul do Brasil. Santa Maria, 2005. 159p.

TEDESCO, M.J.; GIANELLO, C.; BISSANI, C.A.; BOHNEN, H. \& VOLKWEISS, S.J. Análise de solo, plantas e outros materiais. 2.ed. Porto Alegre, Universidade Federal do Rio Grande do Sul, 1995. 174p. (Boletim Técnico, 5)

VELOSO, C.A.C.; GRAÇA, J.J.C. \& GAMA, J.R.N.F Estabelecimento do método DRIS para a cultura de citros na Mesorregião do Nordeste do Estado do Pará. R. Bras. Frutic., 22:372-376, 2000.
WADT, P.G.S. Os métodos da chance matemática e do sistema integrado de diagnose e recomendação (DRIS) na avaliação do estado nutricional de plantios de eucalipto. Viçosa, MG, Universidade Federal de Viçosa, 1996. 123p. (Tese de Doutorado)

WADT, P.G.S.; SILVA, D.J.; MAIA, C.E.; TOMÉ Jr., J.B.; PINTO, P.A. C. \& MACHADO, P.L.O.A. Modelagem de funções no cálculo dos índices DRIS. Pesq. Agropec. Bras., 42:57-64, 2007.

WALWORTH, J.L. \& SUMNER, M.E. The diagnosis and recommendation integrated system (DRIS). Adv. Soil Sci., 6:149-188, 1987.

WALWORTH, J.L.; LETZSCH, W.S.; SUMNER, M.E. Use of boundry lines in establishing diagnostic norms. Soil Sci. Soc. Am. J., 50:123-128, 1986. 